\title{
Introduction to Special Issue on Dualities
}

\author{
Elena Castellani \\ Department of Humanities and Philosophy, University of Florence \\ Dean Rickles \\ Unit for HPS, University of Sydney
}

Key words: Duality, quantum gravity, background independence, holography, emergent spacetime

PACS: 04.50.-h, 11.25.Mj, 11.25.-w, 01.70.+w, 02.40.-k, 11.30.Ly, 11.25.Hf, 11.15.Me

"The concept of duality is crucial in both mathematics and physics."

'Folklore', as quoted in Zeidler (2009, p. 693).

'Duality' is perhaps an overused word in physics and mathematics: there's arithmetic duality, projective duality, Alexander duality, Pontryagin duality, wave-particle duality, electro-magnetic duality, Kramers-Wannier duality, Morita duality, Grothendiek duality, DHS duality, S-duality, T-duality, to name just a handful. It has more than a whiff of the mystical about it, which is rather unfortunate. However, the overhanging mystical elements, especially originating in the concept of 'Yinyang' from Chinese philosophy, are not so off the mark. Like Yinyang, dualities in mathematics and physics are marked by a curious mixture of opposition and sameness or equivalence (see fig.1).

For example, Yin is associated with dark, feminine, passivity, and so on, while Yang is associated with light, masculine, and activity. In Chinese cosmology, the various phenomena of the universe are then viewed as an interplay of these duelling concepts. Further, the two sides are inextricably entangled and interdependent, neither making complete sense without the other: there is no sense of one side of the dual pair being more fundamental or superior. So seems to be the case with dualities in science, with a similar entanglement holding

Email addresses: elena.castellani@unifi.it (Elena Castellani), dean.rickles@sydney.edu.au (Dean Rickles).

1 DR gratefully acknowledges funding from the FQXi and the Australian Research Council (for the award of a Future Fellowship: FT130100466). 


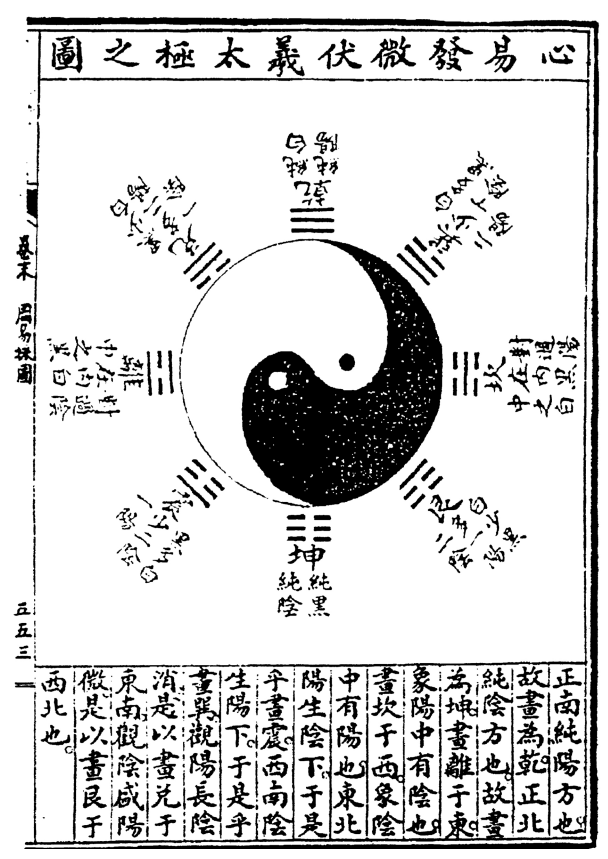

Fig. 1. Taiji diagram (often known as the yinyang diagram). The small white-in-black and black-in-white circles represent the interdependence of opposites - the idea that there is, in some sense, a little of the opposite encoded or embodied in the other. [Image source: Lai Zhide, Yijing Laizhu tujie (Chengdu: Ba Shu shushe, 1688/1989: p. 553).

together duelling concepts such as 'hot/cold,' 'big/small,' 'high energy/low energy,' 'finite/infinite,' 'composite/elementary,' 'localized/delocalized,' etc., though in such a way that an equivalence holds between them at some levelin general, one finds that some quantity and its reciprocal are involved in a duality mapping. There is, then, mystery in dualities, in making sense of how there can be equivalence given such apparently stark differences (differences labeled, in this special issue, as "striking" by De Haro et al. and "shocking" by Huggett). Yet, within this mystery there lies a golden opportunity for philosophers.

Despite their ubiquity and importance in physics, mathematics, and logic, it is fair to say philosophers have yet to embrace dualities with the gusto they deserve. ${ }^{2}$ This is particularly unfortunate since dualities connect to a great many issues that philosophers have fully embraced. To name a few notable examples:

- Reduction, emergence, and fundamentality

- Theoretical equivalence and synonymy

- Underdetermination and empirical equivalence

$\overline{2}$ There are, as always, some exceptions: Dawid (2007), Castellani (2009), Rickles (2011), Matsubara (2013), Muntean (2015), Read (2016). 
- Realism versus anti-realism

- Unification in (and of) mathematics and physics

This special issue, based in part on a conference held in Florence in September 2014, will attempt to bring these connections to wider attention, hopefully functioning as a first point of contact for philosophers previously unacquainted with dualities: dualities promise to enliven, or at least weigh upon, these old philosophers' favourites. What is especially interesting about these above topics, from the point of view of dualities, is the novel ways in which they can be seen to interact. Most of the papers in this volume straddle all of them at once, despite taking some one individual topic as their core focus.

As we understand the concept of duality in this special issue, dualities belong to the general class of symmetries (read 'differences [of one sort] without differences [of another sort]'), though in this case linking theories, or very different regimes of the same theory (in the case of self-duality), rather than solutions to theories as one finds with standard symmetries and gauge symmetries. That is, we should envisage dualities as acting on a space of theories - more precisely, a 'moduli space' - rather than on the space of solutions of a single theory as with standard symmetries. ${ }^{3}$

Because we are transforming one theoretical description to another, the changes are much more radical than with symmetry transformations that map between solutions of the same theory (which will naturally involve entities with the same physical interpretation). Theories, on the other hand, can contain very different natural interpretations in terms of objects (e.g. particles, fields, strings, branes, etc.), properties, degrees of freedom, and spacetime backgrounds that seem to be utterly dissimilar. However, it isn't immediately obvious whether duality transformations point to a representational redundancy or something new. Of course, we can't really answer this question properly until we have some firm principle of individuation for theories, so that we can decide when we have two distinct theories rather than one theory multiply realised or represented. ${ }^{4}$ These and related questions are tackled in several of the essays in this special issue. From this brief description alone, it is clear that the study of dualities combines several central topics of philosophy of physics

3 However, despite this understanding, we have included relevant contributions dealing with a slightly wider notion of duality (such as wave-particle duality and logico-mathematical dualities). These can be brought close enough to our more specific understanding (that of a reciprocal relationship linking seemingly physically incompatible concepts), and because they share many of the same features they have the potential to yield new insights.

4 See Fuchs and Schweigert (1997, p. 14) for more on this point. We might decide ultimately that this is really a distinction without a difference. It is possible that dualities ought to be integral to the debate over how we should individuate theories (something that Rickles discusses in his contribution). 
(and more general philosophy of science) in a novel and interesting way. This special issue will give to readers new to the subject a broad overview of several possible research directions.

The initial essay by Joseph Polchinski, one of the leaders in the development of dualities in string theory, offers a conceptually-oriented guide to the various types of duality in physics: dualities between quantum field theories, between string theories (T-duality and S-duality), and between descriptions which are, respectively, a quantum field theory and a string theory (gauge/gravity dualities). By illustrating the role played by these dualities "in mapping out the structure of theoretical physics" and discussing, at the same time, their conceptual meaning, Polchinski provides an essential overview for an informed and critical reading of the following twelve, more specific contributions to this special issue.

A substantial portion of the philosophical interest in these essays centers on the question of what is meant by 'physical equivalence' (and difference). To obtain from the study of dualities a more nuanced understanding of equivalences between theories and their relationships to one another is surely an important contribution to ongoing philosophical debate. In which sense are dual theoretical descriptions equivalent? Just what kind of equivalence is at stake here? Is the case of dualities different with respect to other more familiar forms of physical equivalences, first of all those related to symmetries in general and gauge transformations in particular? And, finally, does dual equivalence represent a case of underdetermination of theories by empirical data, a traditional issue in the philosophy of science?

Starting from this last question Richard Dawid claims, in his contribution, that a change of perspective on empirical equivalence takes place when it is understood in terms of duality relations. His argument relies on comparing the meaning of dual equivalence in string theory with the "traditional perspective on empirical equivalence" that he identifies as characteristic of 20th century physics: i.e., the view relating the formulation of empirically equivalent theories to "the high degree of flexibility of physical theory building based on advanced mathematics". In this case, the construction of empirically equivalent theories followed, according to Dawid, from an excess of theoretical freedom. In string theory on the contrary, he argues, empirical equivalence is related to "the role of classical limits in a theory that has no free parameters at a fundamental level". As illustrated in Polchinski's introduction to his contribution to this special issue, the presence of dualities in quantum field theories and string theories is related to the fact that a quantum theory may have two or more classical limits: each dual description corresponds to the theory quantized starting from one of these limits and all these descriptions contribute to constructing the full quantum theory. Thus, instead of the redundancy of physical descriptions in terms of which empirical equivalence is 
understood according to the traditional picture, Dawid concludes, duality relations express on the contrary a "mutual inter-dependedness of perspectives, which, on their own, only provide an incomplete understanding".

While Dawid's paper does not problematize the notion of equivalence between scientific theories, a useful characterization of the varieties of equivalence relations between quantum theories is provided by Doreen Fraser in her contribution. In order to illuminate in which sense dual theories can be said to be equivalent, she starts with distinguishing among: predictive equivalence, a map between theories preserving the values of all expectation values and the mass spectra; formal equivalence, a "translation manual" from one theory to the other mapping all quantum states and quantum observables deemed to have physical significance by one theory into quantities in the other theory and respecting predictive equivalence; and physical equivalence, a map between theories respecting both formal and predictive equivalence, and such that each physically significant quantity in the one theory is mapped to a quantity in the other theory with the same physical interpretation. In the light of such distinctions, Fraser's strategy is then to compare and contrast dualities (with a focus on T-duality and mirror symmetry) with the technique of analytic continuation as used in quantum field theory and the status of descriptions of a quantum field theory (relativistic versus Euclidean) related through this technique. This is a particularly useful exercise since, though sharing predictive and formal equivalence, the two cases differ in terms of physical equivalence: while the dual descriptions are judged to be physically equivalent, this is not the case with the descriptions related through analytic continuation. Arguments for the physical equivalence of dual theories must therefore be supported by considerations beyond predictive and formal equivalence, Fraser concludes. Pinpointing the differences with similar but not analogous cases surely helps in clarifying the argument needed to support the claim that the dual theories are physically equivalent. Fraser's essay shows this very effectively.

Sharing the above strategy of investigating the sense in which dual theories are equivalent by comparing with other instructive cases of equivalent theories, Nicholas Teh and Dimitris Tsementzis devote their essay to investigating "the intuitive equivalence" of (hyper-regular) Lagrangian and Hamiltonian classical mechanics. Given the difficulty of finding a satisfactory answer to the question of theoretical equivalence in general, their idea is to develop a fruitful and appealing framework for conceptualizing the cases of theoretical equivalence in physics by starting with analyzing a concrete framework for equivalence in classical mechanics, namely the reformulation of classical mechanics developed in the 1970s by W. M. Tulczyjew. Teh and Tsementzis show how this can be a satisfactory framework for discussing theoretical equivalence, suggesting possible analogies with string dualities. In particular, on the basis of this example they aim to show how concepts and analogies from logic and category theory can be fruitfully employed in the endeavour to understand mathematically 
sophisticated cases of theoretical equivalences.

A similar focus on the role of category theory, and in general meta-mathematical tools, in investigating the nature of dualities can be found in the essay by David Corfield. His paper is a contribution to the dialogue between philosophers of mathematics and philosophers of physics in interpreting the dualities and equivalences relevant to a highly mathematized research field such as current fundamental physics. In particular, Corfield shows how much category theory has to say on the subject (for example, in terms of "arrow reversal, dualising objects and adjunctions"), and how the developments of this research area have vindicated Nagel's 1939 (p. 217) claim that "the concepts of structure, isomorphism, and invariance, which have been fashioned out of the materials to which the principle of duality is relevant, dominate research in mathematics, logic, and the sciences of nature".

Corfield's analysis of the interrelations between the notions of invariance and isomorphism of structure and the form of equivalence implied by dualities in mathematics and physics is closely connected with the philosophical reflections on the relations between dualities and symmetries. Two essays are specifically devoted to comparing dualities with gauge symmetries: De Haro, Teh, \& Butterfield, and Dean Rickles.

In his contribution, Dean Rickles gives some reasons for thinking them identical: all (exact) dualities are simply of the same species as gauge symmetries. The aspects of the dual theories that map onto one another are part of the unphysical, surplus structure only, in much the way that coordinates are unphysical (gauge degrees of freedom) in general relativity, and one 'chooses a gauge' for convenience safe in the knowledge that the (otherwise arbitrary) choice will not affect physical results. This takes some of the mystery out of dualities, and how it is that one can have such radically distinct world-pictures as 10-dimensional and 4-dimensional universes mapping to one another. Underneath this mapping there is preservation of invariant structure: the global symmetries and physical quantities - indeed, one checks for the existence of a duality by focusing on such matching of deep structure. Rickles argues that, from this view it naturally emerges that there must be an invariant theory, with these symmetry properties, that has the dual pictures as representations, in much the way that one can eliminate the gauge freedom from a gauge theory to get the intrinsic theory.

De Haro, Teh, \& Butterfield likewise argue that certain aspects of theoretical structure involved in dualities (such as the radius of a compactified dimension in the context of T-duality) are to be viewed as gauge. However, they also make more precise the nature of the duality relationship itself, highlighting the specific conditions for such mappings (in particular, the mapping is viewed as an isomorphism between theories, or, in more detail, as appropriate bijections 
between the two theories' sets of states and sets of quantities). The important related issue of what exactly is meant by 'theory' here is also carried out. They also elucidate the sense (also mentioned by Polchinski and Rickles in their essays) that some dualities (such as the gauge-gravity duality) are rather curious in that they map gauge symmetries on one side of the duality to gauge symmetries on the other side.

Building on a more general discussion of space in string theory (and the necessity to tease apart distinct senses of 'space'), Huggett puts T-duality to work to argue for the claim that 'phenomenal space' (the broadly non-theoretical space of observable physics) is not fundamental. Huggett focuses in on the perplexing ontological issue of what exactly the physical situation is that underlies a duality, such as a stringy world with one radius as compared to a stringy world with the reciprocal of that radius. Merely saying that they 'describe the same physics' doesn't settle what world-picture we are supposed to form. Huggett argues that the radius is in fact indeterminate in such cases, and draws a parallel with frames of reference in special relativity (and the issue of which is at rest). Just as one can at least envisage using some 'privileged' physical frame (such as the fixed stars) to define preferred rest, so one can imagine using the privileged phenomenal space we observe to 'fix a radius'. Huggett argues that both are equally unmotivated (and, indeed, "reactionary"). Given that T-duals agree on observables (and so the phenomenal space) there must be a mismatch between the string-based space (target space) and phenomenal space, and so the latter cannot be part of the former framework but must instead emerge at a less fundamental level.

As exemplified in Huggett's contribution for the case of T-duality, an effective way to address the philosophical issues arising in relation to the dualities of current fundamental physics is to focus on specific, concrete types of dual equivalence between theories, trying to make the nature of such relationships as precise as possible. Another particularly interesting case study for its apparent ontological implications is weak/strong duality or S-duality (as is usual to call it in the context of string theory). As illustrated in the contribution by Polchinski, this duality type has become a basic ingredient in field and string theories, in particular since the 1990s. In generic terms, it is described as an equivalence map between two different theories 'of the same physics,' where the weak coupling regime of one theory is mapped to the strong coupling regime of the other theory and vice versa. Hence the special interest in this form of duality, seen as a new tool for getting information on physical quantities in the case of large values of the coupling constant, where the usual perturbative methods fail, by exploiting the results obtained in the weak coupling regime of the dual description.

The remarkable relevance of weak/strong duality to recent developments in theoretical physics, as well as its deep connection with important mathemati- 
cal advances, ${ }^{5}$ would be enough to raise the interest of philosophers, especially in regard to such issues as the modalities of theory building and theory assessment in highly mathematized physical research. The story of weak/strong duality in the framework of fundamental physics - starting with the generalization of Dirac's electric/magnetic duality to quantum field theory in the 1970s, to arrive at its current role in string theory - undoubtedly offers rich material for methodological and epistemological investigations. ${ }^{6}$ Systematic studies in such direction have yet to be undertaken, but it surely is only a matter of time. Up to now, the attention of philosophers has focussed substantially on two points: the first one, regarding all physical dualities and thus including weak/strong duality as a special case, is the general issue of theoretical equivalence mentioned above; the second, more specific point concerns a well-known, though surprising feature of weak/strong duality: the fact that, under this sort of duality, it often happens that what is viewed as 'elementary' in one description gets mapped to what is viewed as 'composite' in the dual description and vice versa. At first sight, such interchanging role of 'elementary' and 'composite' seems to have strong implications for reductionism and fundamentality issues. A common claim is that it naturally involves a sort of 'relativity of fundamentality': what kinds of things are considered to be fundamental depends on the dual description considered. The question is whether it is legitimate to assume that there are implications of this sort, and, in case, in what the impact of weak/strong duality on these and other related philosophical issues really consists.

Kerry McKenzie, in her contribution, addresses the second side of this question by carefully analyzing the implications of S-duality for the metaphysics of fundamentality. She argues that the "relativity of fundamentality inherent in S-duality represents something genuinely new" with respect to other familiar cases of relative fundamentality, and shows how this relativity has both realist and anti-realist implications. In particular, she critically discusses the argument, defended in previous philosophical literature, ${ }^{7}$ according to which envisaging some form of ontological structural realism (in short, the thesis that "all there is, is structure") is the only option viable for escaping the antirealist conclusions apparently implied by the elementary/composite ambiguity. She shows that "there is no obvious route from S-duality to ontic structural realism", and discusses, more in general, what the significance of S-duality can be for structuralist metaphysics.

The first side of the question is discussed in the essay by Elena Castellani. Her contribution follows an 'integrated' approach, by analyzing the meaning of the elementary/composite correspondence in the light of the history of

\footnotetext{
5 See, on these connections, Corfield's contribution and references therein.

6 For a brief outline of this history, see Castellani's essay in this volume, section 2.

7 See Dawid (2007), Rickles (2011), and Matsubara (2013).
} 
weak/strong duality. On this aim, a substantial part of her paper is devoted to reconstruct the main developments of the idea of electric-magnetic duality from which the weak/strong duality type originates - from Dirac's theory of magnetic monopoles to the successive generalizations of electric-magnetic duality in the context of (Abelian and non-Abelian) field theory and its first extension to string theory. This historical analysis is then used as evidential basis for discussing the philosophical implications of weak/strong duality. Castellani's conclusion is that what this duality type specifically implies, in the cases considered, regards not so much (or not always) mutual composition of the particles of the theory (as the elementary/composite correspondence can misleadingly suggest) as rather their different modes of appearance when considering the different classical limits of the quantum theory, i.e. the dual perspectives: the particles interchangeably play the role of Noether charges or topological charges depending on the perspective under which the theory is considered. The philosophical lesson to be drawn from this sort of "particle democracy', ${ }^{8}$ she claims, is that the different characterizations of the particles of the theory - as elementary particles or as solitons, depending on the particular formulations of the theory in certain regions of its parameter space in the case of generalized electric-magnetic duality -, should not be taken too literally.

To avoid a literal reading of the different characterizations of the dual counterparts is a shared attitude, in this volume. It corresponds to the idea that what the dual descriptions do not agree upon, should not be attributed a real physical significance. ${ }^{9}$ An already mentioned case is Huggett's analysis of how to understand the interchange of tiny and huge dimension connected with T-duality in string theory. Another example is the interpretation of dual correspondences in the case of gauge/gravity duality which is provided in the contribution by Sebastian de Haro to this volume. His essay is devoted to analyzing the viability of this type of duality - a duality between a theory of gravity (defined in a 'volume' bounded inside a given surface) and a quantum field theory (defined on that surface) - as a particular approach to quantum gravity. On this aim, he considers the cases of AdS/CFT, black holes and Verlinde's scheme, and discusses whether conditions for a theory of gravity such as background independence are satisfied. In this discussion, the interpretation he argues for is what he calls an "internal interpretation" (following the distinction between an external and an internal point of view introduced in Dieks, van Dongen and de Haro, 2015). This is the interpretation according to which there is no external context fixing the meaning of the physical quantities

\footnotetext{
8 This is the expression used by Ashoke Sen, one of the leading physicists working in the field who has been particularly attentive to the possible implications of this sort of dual correspondence.

${ }^{9}$ In this sense some authors propose to view duality as a 'gauge'. See Rickles's contribution, in particular.
} 
related through the duality: the two dual descriptions agree on everything that is physically meaningful and the interpretation of the dual correspondents is not fixed beforehand. For example, in the framework of AdS/CFT, ${ }^{10}$ what might be interpreted as a 'length' in the first description, can be reinterpreted in terms of 'renormalisation group scale' in the dual description, as illustrated by de Haro.

Given that, according to this interpretation, the two sides of the duality should be taken as physically equivalent representations of the same theory, emergence of one component (gravity) from the other (gauge theory) would seem to be excluded. However, the issue of the relation between dualities and emergence is a subtle one, and de Haro's essay is a contribution to discussing it, both in general and in the specific case of gauge/gravity duality. More precisely, by examining the conditions under which emergence of gravity can take place, de Haro argues that emergence of gravity "can only relate to approximate dualities, the duality being modified by coarse graining".

The essay by Tiziana Vistarini is a further contribution to the philosophical reflection on the relation between dualities and emergence. Like Huggett, she too attempts to say something about the deeper metaphysical claims (vis-àvis spacetime ontology) underlying dualities. She carefully detaches emergent spacetime claims from duality symmetries by focusing on standard philosophical conceptions of emergence, highlighting where crucial incompatibilities occur. Vistarini suggests that a 'double-aspect monism' (according to which one and the same underlying structure presents in multiple distinct, though nonetheless 'real' modes) offers the best prospect for a metaphysics of dualities. This view respects the absence of a fundamental side of a duality while allowing a thin notion of emergence (which she associates with spacetime structure not being reducible to degrees of freedom internal to the dual theory to which it belongs).

Closing this special issue, the contribution by Peter Bokulich goes full circle back to the starting point by focusing on wave-particle duality (the first example mentioned in the initial essay by Polchinski for illustrating the meaning of a duality in quantum theories, i.e. the idea that the presence of a duality is related to the fact that the quantum theory has two different classical limits). Quoting from Polchinski's introductory section: "given a quantum field theory, one can take two different classical limits depending on what one holds fixed. One limit gives classical fields, the other classical particles." At this point, a natural question is how much this kind of wave-particle duality can be related to the original notion introduced in the context of non-relativistic quantum

\footnotetext{
$\overline{10}$ Maldacena's duality between a string theory (gravity theory) on an anti-de Sitter spacetime (AdS) and a gauge theory (a conformal field theory, CFT) on its boundary.
} 
mechanics. This is the question specifically addressed by Bokulich. His contribution is in fact an attempt to clarify the different meanings of wave-particle duality, starting with the notion of Bohr and its relation with complementarity. Bokulich argues for a careful distinction among wave-particle duality and what Bohr meant by complementarity. He also claims that there are some crucial obstacles, having to do with the measurement problem, to linking Bohr's notion of wave-particle duality with the modern conception of duality, e.g., as outlined in Polchinski's essay. Nonetheless, bringing wave-particle duality under the rubric of dualities as intended in this issue has the potential to impact upon the way wave-particle duality (or the related/conflated notion of 'complementarity') is conceptualised.

\section{References}

Fuchs, J. and C. Schweigert (1997), Symmetries, Lie Algebras and Representations: A Graduate Course for Physicists. Cambridge University Press.

Zeidler, E. (2009) Quantum Field Theory I: Basics in Mathematics and Physics. Springer.

Castellani, E.(2009).Dualities and intertheoretic relations.In: M. Surez, M. Dorato, \& M. Redei (eds.), EPSA philosophical issues in the sciences: Launch of the European philosophy of science association, (pp. 919). Springer.

Dawid, R. (2007). Scientific Realism in the Age of String Theory. Physics and Philosophy, 11, 1863-7388.

Matsubara, K. (2013). Realism, underdetermination and string theory dualities. Synthese, 190 (3), 471-489.

Muntean, I. (2015). Metaphysics From String Theory: S-Dualities, Fundamentality, Modality and Pluralism. In T. Bigaj \& ChWuthrich (eds.), Metaphysics in Contemporary Physics. Brill/Rodopi 259292.

Read, J. (2016) The Interpretation of String-Theoretic Dualities. Foundations of Physics, 46, 209-235.

Rickles, D. (2011). A philosopher looks at string dualities. Studies in History and Philosophy of ModernPhysics, 42(1), 54-67. 